\title{
Diamagnetic susceptibility of spin-triplet ferromagnetic superconductors
}

\author{
H. Belich ${ }^{1,2}$, Octavio D. Rodriguez Salmon ${ }^{1}$, Diana V. Shopova ${ }^{3}$, \\ and Dimo I. Uzunov ${ }^{1,3 \dagger}$ \\ ${ }^{1}$ International Institute of Physics, Universidade Federal de Rio Grande do Norte, \\ av. Odilon Gomes de Lima, 1722, 59078-400, Natal (RN), Brazil. \\ ${ }^{2}$ Universidade Federal do Espírito Santo (UFES), Departamento de Fúsica e \\ Química, Av. Fernando Ferrari 514, Vitória, ES, CEP 29075-910, Brazil. \\ ${ }^{3}$ Collective Phenomena Laboratory, G. Nadjakov Institute of Solid State Physics, \\ Bulgarian Academy of Sciences, BG-1784 Sofia, Bulgaria. \\ † Corresponding author: d.i.uzunov@gmail.com
}

Key words: Ginzburg-Landau theory, thermodynamic property, superconductivity, ferromagnetism, magnetization, phase diagram.

PACS: 74.20.De, 74.20.Rp

\begin{abstract}
We calculate the diamagnetic susceptibility in zero external magnetic field above the phase transition from ferromagnetic phase to phase of coexistence of ferromagnetic order and unconventional superconductivity. For this aim we use generalized Ginzburg-Landau free energy of unconventional ferromagnetic superconductor with spin-triplet electron pairing. A possible application of the result to some intermetallic compounds is briefly discussed.
\end{abstract}

In certain ferromagnetic unconventional superconductors the phase transition to superconductivity states occurs in the domain of stability of ferromagnetic phase (an example is the itinerant ferromagnet $\mathrm{UGe}_{2}$ [1, 2, 3]). This seems to be a general feature of ferromagnetic superconductors with spin-triplet electron pairing [4, 5, 6] (see also reviews [7, 8]). In such situation the thermodynamic properties near the phase transition line may differ from those known for the superconducting-to-normal 


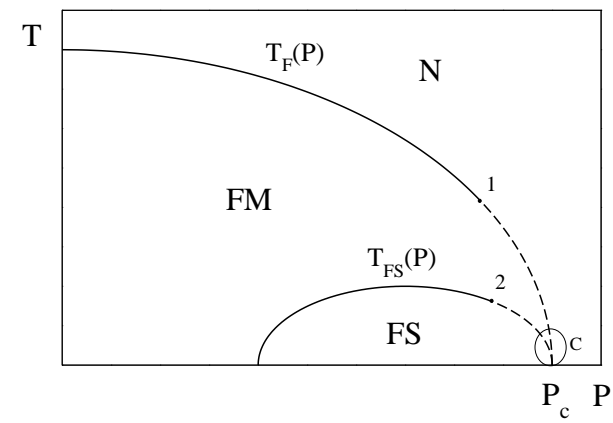

Figure 1: An illustration of the $T-P$ phase diagram of $\mathrm{UGe}_{2}$ (details are omitted): $\mathrm{N}$ - normal phase, FM - ferromagnetic phase, FS - phase of coexistence of ferromagnetic order and superconductivity, $T_{F}(P)$ and $T_{F S}(P)$ are the respective phase transition lines (solid line corresponds to second order phase transition, dashed lines correspond to first order phase transitions; 1 and 2 are tricritical points; $P_{c} \sim 1.6 \mathrm{GPa}$ is the critical pressure; $T_{F}(0) \sim 53 \mathrm{~K} ; T_{F S}<1.22 \mathrm{~K}$; the loop C indicates a small domain $(T<0.3 \mathrm{~K}, P \sim 16 \mathrm{GPa})$ where the shape of the phase diagram is not well established by available experimental data.

metal transition. We show this by using the example of diamagnetic susceptibility above the phase transition line of superconducting transition in spin-triplet ferromagnetic superconductors. This is the line in the temperature-pressure $(T-P)$ phase diagram (Fig. 1), which separates the pure ferromagnetic phase (FM) and the phase (FS) of coexistence of ferromagnetic order and superconductivity. Here we present the result for diamagnetic susceptibility which follows from the GinzburgLandau theory for such type of superconductors [4, 5, 6]. We outline the main steps of calculation of diamagnetic susceptibility in the Gaussian approximation. At the end we briefly discuss the possible application of our results to real systems.

Following notations and results in Refs. [4, 7, 8], we present the GL free energy (fluctuation Hamiltonian) of spin-triplet ferromagnetic superconductors, which is essential in the present consideration, namely

$$
\mathcal{H}=\int d^{3} x\left\{\hat{H}_{0}[\psi(\mathbf{x})]+\hat{H}_{M}[\psi(\mathbf{x})]\right\}
$$

by the energy densities

$$
\hat{H}_{0}=\frac{\hbar^{2}}{4 m} \sum_{j=1}^{3}\left|\left(\nabla-\frac{2 i e}{\hbar c} \mathbf{A}\right) \psi_{j}\right|^{2}+a_{s}|\psi|^{2}
$$

and 


$$
\hat{H}_{M}=i \gamma_{0} \mathbf{M} \cdot\left(\psi \times \psi^{*}\right)+\rho \mathbf{M}^{2} \cdot \psi^{2}
$$

In Eqs. (2)-(3),$\psi(\mathbf{x})=\left\{\psi_{j}(\mathbf{x}) ; j=1,2,3\right\}$ is three dimensional vector field with complex components $\psi_{j}$, which represents the superconducting order, $\mathbf{M}$ is the spontaneous magnetization, the vector potential $\mathbf{A}$ is related to the magnetic induction by $\mathbf{B}=\mathbf{H}+4 \pi \mathbf{M}$ and obeys the Coulomb gauge $(\nabla \cdot \mathbf{A}=0) ; a_{s}=\alpha_{s}\left(T-T_{s}\right), \gamma_{0}$ and $\rho$ are positive material parameters, and $2 e$ and $2 m$ are the charge and the effective mass of the electron Cooper pairs, respectively. We neglect the possible spatial anisotropy, which is usually represented in the gradient terms of the Hamiltonian $\mathcal{H}$ (see, e.g., Ref. [4, 7, 8]).

Our task is to calculate the equilibrium free energy

$$
F=-\beta^{-1} \ln \int \prod_{\mathbf{x} \in V} \mathcal{D} \psi(\mathbf{x}) \exp (-\beta \hat{H}),
$$

in the volume $V=L_{x} L_{y} L_{z}$ of the superconductor and the diamagnetic susceptibility per unit volume in zero external magnetic field, given by $\chi=\left[-\partial^{2} F / V \partial^{2} H\right]_{H=0}$; $\beta^{-1}=k_{B} T$. In Eq. (4), the functional integral is taken over both real $[\Re \psi(\mathbf{x})]$ and imaginary $[\Im \psi(\mathbf{x})]$ parts of the complex field $\psi(\mathbf{x})$, i.e., $\mathcal{D} \psi(\mathbf{x}) \equiv d \Re \psi(\mathbf{x}) d \Im \psi(\mathbf{x})$. Note that for temperatures near $T_{F S}(P)$ we can always set $\beta \approx \beta_{F S}=1 / k_{B} T_{F S}$ (see, e.g., [9]).

As far as the behaviour in FM phase in a close vicinity of curve $T_{F S}(P)$ is of interest to our consideration, (see Fig. (1), the magnetization $M$ has a magnitude $|\mathbf{M}| \equiv M$, given by $M(T, P)=\left[\alpha_{f}\left(T-T_{F}\right) / b_{f}\right]^{1 / 2}$, i.e., the result from the standard Landau theory of ferromagnetic transitions with parameters $a_{f}=\alpha_{f}\left(T-T_{F}\right)$ and $b_{f}$ [4]

$$
F_{m}=a_{f} \mathbf{M}^{2}+\frac{b_{f}}{2} \mathbf{M}^{4}
$$

where $a_{f}=\alpha_{f}\left(T-T_{F}\right)$, and $b_{f}>0$. Therefore, in our consideration $M(T, P)$ is a known thermodynamic quantity, which is established by the exhaustive thermodynamic analysis of the phases in the unconventional superconductor in [4].

We choose the magnetization $\mathbf{M}=(0,0, M)$ and the external magnetic field $\mathbf{H}=$ $(0,0, H)$ to lie along the $\hat{z}$-axis. Then the first term in Eq. (3) takes the simple form $M\left(\psi \times \psi^{*}\right)_{z}=M\left(\psi_{1} \psi_{2}^{*}-\right.$ c.c. $)$. Under the supposition of uniform external magnetic field $H$, we take the gauge of the vector potential $\mathbf{A}$ as $\mathbf{A}=(-B y, 0,0)$, and following classic papers [10, 11, 12], we can represent the fields $\psi_{j}(\mathbf{x})$ by the series 


$$
\psi_{j}(\mathbf{x})=\frac{1}{L_{x} L_{z}} \sum_{q} c_{j}(q) \varphi_{j}(q, \mathbf{x})
$$

in terms of the eigenfunctions

$$
\varphi_{j}(\mathbf{q}, \mathbf{x})=\frac{1}{\left(L_{x} L_{z}\right)^{1 / 2}} e^{i\left(k_{x}+k_{z}\right)} u_{n}(y)
$$

of the operator $[i \hbar \nabla+(2 e / c) \mathbf{A}]^{2} / 4 m$, corresponding to the eigenvalues

$$
E(q)=\left(n+\frac{1}{2}\right) \hbar \omega_{c}+\frac{\hbar^{2}}{4 m} k_{z}^{2}
$$

specified by the quantum number $n=0,1, \ldots, \infty$, the wave vector components $k_{x}$ and $k_{z}$, and the cyclotron frequency $\omega_{c}=(e B / m c)$. In Eq. (6) $)$, the function $u_{n}(y)$ is related to the Hermite polynomials $H_{n}(y)$ by

$$
u_{n}(y)=A_{n} e^{-\frac{\left(y-y_{0}\right)^{2}}{2 a_{H}^{2}}} H_{n}\left(\frac{y-y_{0}}{a_{H}}\right),
$$

where $A_{n}^{-1}=\left(a_{B} 2^{n} n ! \sqrt{\pi}\right)^{1 / 2}$ [13], $y_{0}=a_{B}^{2} k_{x}$, and $a_{B}=(\hbar c / 2|e| B)^{1 / 2} ; B=|\mathbf{B}|$.

Now the fluctuation Hamiltonian becomes $\mathcal{H}=\sum_{q} \hat{\mathcal{H}}(q)$ with

$$
\begin{aligned}
\hat{\mathcal{H}}(q) & =\sum_{j} \tilde{E}(q) c_{j}(q) c_{j}^{*}(q) \\
& +i \gamma_{0} M\left[c_{1}(q) c_{2}^{*}(q)-\text { c.c. }\right]
\end{aligned}
$$

where

$$
\tilde{E}(q)=E(q)+a_{s}+\rho M^{2}
$$

Applying the unitary transformation,

$$
\begin{aligned}
& c_{1}(q)=\frac{i}{\sqrt{2}}\left[-\phi_{+}(q)+\phi_{-}(q)\right] \\
& c_{2}(q)=\frac{1}{\sqrt{2}}\left[\phi_{+}(q)+\phi_{-}(q)\right]
\end{aligned}
$$

renders the fluctuation Hamiltonian as a sum of squares of field components $c_{3}(q)$, and $\phi_{ \pm}(q)$, and the free energy (44) can be calculated as usual Gaussian integrals over the same fields. 
Following approximations, justified in Ref. [12], we obtain the result

$$
\frac{F}{V}=\mu B^{2}\left(\frac{1}{a_{-}^{1 / 2}}+\frac{1}{a_{0}^{1 / 2}}+\frac{1}{a_{+}^{1 / 2}}\right)
$$

where

$$
a_{ \pm}\left(\gamma_{0}\right)=a_{s}+\rho M^{2} \pm \gamma_{0} M
$$

$a_{0} \equiv a_{ \pm}(0)$, and $\mu=e^{2} k_{B} T / 24 \pi \hbar c^{2} m^{1 / 2}$. Having in mind that $\partial / \partial H=\partial / \partial B$, the fluctuation diamagnetic susceptibility in Gaussian approximation takes the form

$$
\chi(T)=-2 \mu\left(\frac{1}{a_{-}^{1 / 2}}+\frac{1}{a_{0}^{1 / 2}}+\frac{1}{a_{+}^{1 / 2}}\right),
$$

In contrast to usual superconductors [12], where the contribution to the free energy from the diamagnetic currents is represented by a single term, here we have three terms with labels 0 , and \pm which exactly correspond to the contributions of the field components $c_{3}$, and $\phi_{ \pm}$, respectively.

Now one should use known results [4, 5, 6, 7, 8, to analyze the singularities of free energy in a close vicinity $\left(0<T-T_{F S} \ll T_{F S}\right)$ to the phase transition curve $T_{F S}(P)$ in the FM phase $\left(T_{F}>T>T_{F S}\right)$, where $M(T, P)=\left[\alpha_{f}\left(T_{F}-T\right) / b_{f}\right]^{1 / 2}$ and, for some real intermetallic compounds, for example, $\mathrm{UGe}_{2}$, the condition $\left(T_{F}-T_{F S}\right) \gg$ $\left(T-T_{F S}\right)$ is satisfied. We shall briefly discuss the behaviour of the free energy (13) near the left-hand part of the curve $T_{F S}(P)$, where the phase transition FM-FS is of second order. For this case the critical temperature $T_{F S}(P)$ is given in Refs. [5, 6]. In the present notations $T_{F S}(P)$ is defined by the equation

$$
T_{F S}=T_{s}-\frac{\rho}{\alpha_{s}} \Delta+\frac{\gamma_{0}}{\alpha_{s}} \Delta^{1 / 2}
$$

where $\Delta \equiv\left[M\left(T_{F S}\right)\right]^{2}=\alpha_{f}\left(T_{F}-T_{F S}\right) / b_{f}>0$. Expanding $a_{0}(T)$, and $a_{ \pm}\left(\gamma_{0}, T\right)$ to first order in $\left(T-T_{F S}\right)$, one may easily check that $a_{-}\left(T_{F S}\right)=0$ and

$$
a_{-}(T) \approx \tilde{a}_{-}\left(T-T_{F S}\right)
$$

where

$$
\tilde{a}_{-}=\alpha_{s}-\frac{\rho \alpha_{f}}{b_{f}}+\frac{\gamma_{0} \alpha_{f}^{1 / 2}}{2\left[b_{f}\left(T_{F}-T_{F S}\right)\right]^{1 / 2}},
$$


while $a_{0}$ and $a_{+}$remain positive at $T_{F S}: a_{0}\left(T_{F S}\right)=\gamma_{0} \Delta^{1 / 2}$, and $a_{+}\left(T_{F S}\right)=2 a_{0}\left(T_{F S}\right)$. Therefore, only one of all three fluctuation diamagnetic contributions in Eqs. (13) and (15) will generate singularity of the free energy and the typical divergence of susceptibility. Keeping only the singular term in Eq. (13), we obtain that in a close vicinity of line $T_{F S}(P)$, where $a_{-} \ll \min \left(a_{0}, a_{+}\right)$,

$$
\chi(T)=\frac{\chi_{0}}{\left(T-T_{F S}\right)^{1 / 2}},
$$

$\left(T>T_{F S}\right)$, where the scaling amplitude $\chi_{0}$ is given by

$$
\chi_{0}=-\frac{2 \mu}{\tilde{a}_{-}^{1 / 2}} .
$$

Note that $a_{ \pm}\left(\gamma_{0}\right)>0$ is a condition for the stability of the FM phase and, therefore, the quantity $\tilde{a}_{-}$is always positive for $T_{F S}(P)<T<T_{F}(P)$.

The formulae (19a) and (19b) are our main result. This scaling relation [9] is of typical Gaussian type with an inverse root dependence on $\left(T-T_{F S}\right)$ whereas the scaling amplitude $\chi_{0}$ contains an essentially new information. Compared to known result for usual superconductors [12, the fluctuation diamagnetic susceptibility (19a) contains an extra factor $\left(\tilde{a}_{-}\right)^{-1 / 2}$, which depends on the material parameters of the unconventional ferromagnetic superconductor. The value of the new susceptibility amplitude factor $\left(\tilde{a}_{-}\right)^{-1 / 2}$ in Eq. (19b) should be taken at $T_{F S}(P)$ for any pressure $P$ of interest. Thus in evaluating the parameter $\tilde{a}_{-}$we may use the Eq. (16) for $T_{F S}(P)$.

In some real systems the Eq. (18) can be simplified. For example, in $\mathrm{UGe}_{2}, T_{s} \sim 0$ $\mathrm{K}$ [5, 6], $T_{F} \gg T_{F S}$ [1] and, therefore, one may use $\tilde{a}_{-} \approx\left(\alpha_{s}-\rho \alpha_{f} / 2 b_{f}\right)$. This result is obtained with the help of Eq. (16). In itinerant ferromagnets with uniaxial anisotropy as, for example, $\mathrm{UGe}_{2}$, both phases FM and FS may occur in two domains with opposite magnetizations $|\mathbf{M}|= \pm M$. Here we have considered FM and FS with $M>0$. In the domains of FM, where $M<0$, the singular parts of the free energy and the susceptibility will be given by the terms, containing the quantity $a_{+}$. Because of the invariance of the Eqs. (13) and (15) with respect to the change $a_{ \pm} \rightarrow a_{\mp}$, the results presented by Eqs. (13), (15), and (19a)-(19b) are valid in both domains of the FM and $\psi$-fluctuations corresponding to any domain $(M \lessgtr 0)$ of FS [4].

We have used the Gaussian approximation, which is not valid in the critical region [9] of anomalous fluctuations. However, the critical region of real ferromagnetic superconductors with spin-triplet electron pairing is often very narrow and, 
hence, virtually of no interest. Therefore, the present results can be reliably used in interpretation of experimental data for real itinerant ferromagnets, which exhibit low-temperature spin-triplet superconductivity triggered by the ferromagnetic order.

\section{References}

[1] S. S. Saxena, P. Agarwal, K. Ahilan, F. M. Grosche, R. K. W. Haselwimmer, M.J. Steiner, E. Pugh, I. R. Walker, S.R. Julian, P. Monthoux, G. G. Lonzarich, A. Huxley. I. Sheikin, D. Braithwaite, and J. Flouquet, Nature (London) 406 (2000) 587.

[2] A. Huxley, I. Sheikin, E. Ressouche, N. Kernavanois, D. Braithwaite, R. Calemczuk, and J. Flouquet, Phys. Rev. B 63 (2001) 144519.

[3] N. Tateiwa, T. C. Kobayashi, K. Hanazono, A. Amaya. Y. Haga. R. Settai, and Y. Onuki, J. Phys. Condensed Matter 13 (2001) L17.

[4] D. V. Shopova, and D. I. Uzunov, Phys. Lett. A 313 (2003) 139; Phys. Rev. B 72 (2005) 024531.

[5] M. G. Cottam, D. V. Shopova, and D. I. Uzunov, Phys. Lett. A 373 (2008) 152.

[6] D. V. Shopova and D. I. Uzunov, Phys. Rev. B 79 (2009) 064501.

[7] D. V. Shopova, and D. I. Uzunov, Bulg. J. Phys. 32 (2005) 81.

[8] D. V. Shopova and D. I. Uzunov, in: Progress in Ferromagnetism Research, ed. by V. N. Murray (Nova Science Publishers, New York, 2006) pp. 223. ISBN: 1-59454-469-7.

[9] D. I. Uzunov, Introduction to the theory of critical phenomena (World Scientific, Singapore, 1993); 2nd Edition: World Scientific, New Jersey, 2010.

[10] H. Schmidt, Z. Physik 216 (1968) 336.

[11] A. Schmid, Phys. Rev. 180 (1968) 527.

[12] E. M. Lifshitz and L. P. Pitaevskii, Statistical Physics, Part II (Pergamon, London, 1980).

[13] M. Abramowitz and I. A. Stegun (eds.), Handbook of Mathematical Functions (Dover, New York, 1965). 